\title{
Impact of Mobility in Ad Hoc Protocol Design
}

\author{
Arta Doci \\ Colorado School of Mines \\ adoci@mines.edu
}

\author{
Georgios Samakovitis \\ New York College, Athens \\ gsamakovitis@nyc.gr
}

\author{
Vijaya Raju \\ University of New York, Tirana \\ vrmullagiri@unyt.edu.al
}

\begin{abstract}
Protocols in ad hoc networks are not designed with mobility in mind. Recent research reveals that mobility impacts all the layers of the protocol stack. Specifically, more realistic mobility models that are extracted from real user traces for the vehicular and pedestrian scenarios show that wireless nodes tend to cluster around popular locations. The contributions of this paper are two-fold. First, it suggests cross layer design, as a promising approach, in designing ad hoc protocols with mobility in mind. Therefore, it provides a survey of the methodologies used in wireless cross layer studies. Second, it presents a framework for cross layer and flexible ad hoc protocol design, which integrates mobility into protocol design.
\end{abstract}

\section{INTRODUCTION}

The goal of any wireless network is to maximize its overall performance. In order to do so we need to have a thorough knowledge of the factors from each layer that affect the performance. For example, "mobility impacts the entire protocol stack of a communication network with mobile nodes"[6]; yet, mobility has not been made part of the protocol design. In addition, a comprehensive list of factors that need to be exposed across layers is still missing. For example, mobility impacts traffic[4], which depends on the access point that it is associated with; therefore, physical layer could expose to the routing layer the traffic flow and other pertinent information. This suggests that cross layer design could be a promising alternative, when taking mobility into account.

Cross layer design enables improved overall network performance and increased network lifetime [5, 8, 23, 25]. Separate cross layer design surveys for cellular [10], ad hoc [19], and sensor networks [11] have been provided. These surveys and most of the research on cross layer interaction design in wireless networks $[18,13,12,15]$ use analytical models to provide cross layer interaction solutions.

In this paper we survey the factor selection methods in wireless networks by classifying these methods around three main groups. The taxonomy is provided by grouping the methods around the following three questions that need to be answered: (1) What factors ${ }^{1}$ from each layer should be considered when studying cross layer interactions?; (2) What factor interactions affect an observed performance?; and (3) Which combinations of levels of factors maximize network performance?

Furthermore, real mobility models for pedestrian [24] and vehicular [1] reveal that nodes tend to cluster around popular locations. Using this information from mobility models it appears that flexible ad hoc protocols are more suitable then reactive protocols, ,i.e., [16] and [3]. A flexible protocol should posses the following:

- Automatically assess the appropriate number of clusters that the protocol should adjust to operate.

- Adopt a protocol that is better suited for stationary nodes (table-driven, when within clusters).

- Adjust dynamically to a protocol that is less vulnerable to mobility, when between clusters (AODV)

In this paper we focus to address only the first item of the list provided in the previous paragraph, since it has not been addressed so far. While it is well known that some AP's handle most of the traffic, than it is important to assess the number of clusters based on the traffic handled, as well as, cluster size. In addition, in this paper we provide a taxonomy and propose a framework for flexible ad hoc protocols in cross layer design settings.

\section{WHAT FACTORS FROM EACH LAYER SHOULD BE CONSIDERED WHEN STUDYING cross layerINTERACTIONS?}

Historically, the factors considered from each layer in cross layerdesign have been selected by experts on what

\footnotetext{
${ }^{1} \mathrm{~A}$ factor is a parameter that affects the execution of a given protocol.
} 
is believed to be relevant based on experience. For example, in [20] the authors discuss important physical layer factors, such as signal reception, path loss, fading, interference, noise computation, and preamble length, which have an impact on the performance of routing protocols for generic ad hoc networks.

We believe that the only research, which has evaluated a large number of factors with a formal methodology is [9]. The authors used stepwise regression and best subsets regression as the factor selection methods for selecting factors that substantially affected delivery ratio. In this section, we describe both stepwise and best subset regression methods.

\subsection{Stepwise Regression}

The goal of stepwise regression is to determine a subset of factors that most influence the performance metric, e.g., the delivery ratio in this study. There are three methods that can be used, namely forward selection, backward elimination, and a hybrid of the two. The forward selection method begins with no factors in the model. For each factor, the $F$ statistic is calculated. The method uses a threshold value for the lowest possible $F$, usually called $F_{i n}$, which determines if any factor is significant enough to start building a model. The $p-$ value for each $F$ statistic is compared to $F_{\text {in }}$ (or to the default value of 0.50 ). If no $F$ statistic has a $p$-value less than $F_{i n}$, then the method stops. Otherwise, the method adds the factor that has the smallest $p$-value less than $F_{\text {in }}$ (i.e., the largest $F$ statistic). The method then calculates $F$ statistics for the factors remaining outside the model, and the evaluation process is repeated. Thus, factors are added one by one to the model until no remaining factor produces a significant $F$ statistic.

Backward elimination works in the opposite direction of forward selection; this method starts the model loaded with factors and tries to reduce these factors down to relevant ones. It begins by calculating an $F$ statistic for each factor in the model. The method uses a threshold value called $F_{\text {out }}$. The $p$-value for each $F$ statistic is compared to $F_{\text {out }}$ (or to the default value of 0.1 ). The factors are then deleted from the model one by one until all the factors remaining in the model produce $F$ statistics significant at the threshold $F_{\text {out }}$. At each step, the factor with the smallest contribution to the model (,i.e., with the smallest $F$ statistic or equivalently the largest $p-$ value) is deleted.

The hybrid method starts by adding a new factor via the forward selection method. When necessary, one of the factors is then removed via backward elimination. Two threshold $F$-values ( $F_{\text {in }}$ and $F_{\text {out }}$ ) are defined such that $F_{\text {in }}>F_{\text {out }}$, making it more difficult to add factors to the model than to delete factors from the model.

\subsection{Best Subsets Regression}

This method calculates all possible regression models containing one, two, three, etc. factors and then chooses the best regression model. Consider the following linear regression model with three factors $X_{1}, X_{2}$ and $X_{3}$ :

$$
Y=a+b_{1} X_{1}+b_{2} X_{2}+b_{3} X_{3}+e=a+\sum_{i} X_{i} b_{i}+e,
$$

where $i$ represents the number of factors, $a$ is the intercept, $b_{i}$ is the effect of $X_{i}$ on $Y$, and $e$ is the error of the regression model. We can fit $2^{3}-1=7$ possible regression models. Now, suppose we have $p$ factors. Then, we can fit $2^{p}-1$ possible regression models. A natural question that arises is: what criteria should we use to select the best regression model? The most common criterion is the coefficient of multiple determinations, which is denoted by $R^{2}$ [14]. That is, the model with the smallest $R^{2}$ value is determined to be the best regression model

\section{WHAT FACTOR INTERACTIONS AFFECT AN OBSERVED PERFOR- MANCE?}

Statistically defined, there is an interaction between two factors if the effect of one factor depends on the levels ${ }^{2}$ of the second factor. The popular formal methodologies used to detect interactions in wireless networks are design of experiments (DoE) and dependency graphs.

\subsection{DoE}

In [11] and $[22,17,21]$ the authors use DoE to characterize and quantify interactions between layers. The process of DoE includes choosing the factors to include in the study, the levels of the factors, and the performance metric(s) in order to select an appropriate design for the experiment. The most used design method in these studies is factorial design. We note a second less used design method is fractional design (see [2] for an example).

In factorial design, all possible factors and their interactions are tested. Factorial designs are described in regard to the number of levels associated with the factors, i.e., $2 x 3$ factorial design specifies that two levels for the first factor and three levels for the second factor. In order to test for significant factors and interactions in a factorial design, the analysis of variance (ANOVA) method is used.

In mathematical form the linear model of ANOVA is:

$$
y_{i j k}=\mu+\alpha_{i}+\beta_{j}+(\alpha \beta)_{i j}+\varepsilon_{i j k},
$$

\footnotetext{
${ }^{2}$ The different values of a factor are called levels.
} 
where $\mu$ is the overall mean, $\alpha_{i}$ is the effect of factor $A$ ( $i$ represents the level of factor $A), \beta_{j}$ effect of factor $B(j$ represents the level of factor $B),(\alpha \beta)_{i j}$ is the interaction of factors $A$ and $B$, and $\varepsilon_{i j k}$ is the error term ( $k$ represents the number of replications at each combination of levels for factor $A$ and factor $B$ ). In order to judge the amount of interactions an $F$ statistic is generated.

Let's assume we have two factors $A$ and $B$ with two levels each as shown in Table I. One useful way to depict interaction between two factors is with an interaction plot as shown in Figure 1. When interactions are present, a graph with non parallel lines is created in an interaction plot (as shown in Figure 1). If the lines are parallel then there is no interaction.

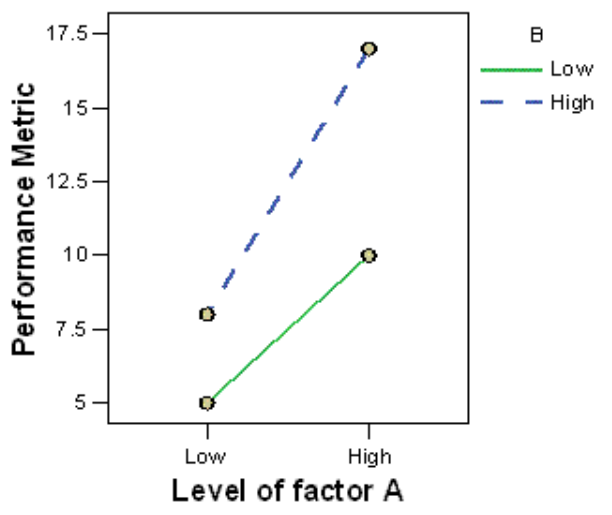

Figure 1. Interaction Plot on two factors $A$ and B.

\subsection{Dependency Graphs}

The authors of [2] propose using dependency graphs to understand possible interactions. This method is sketched as follows. First, represent the parameters of a protocol graphically showing the flow of the parameters. In this dependency graph, every parameter is a node and a directed edge indicates the dependency relation between the parameters. By combining graphs of various protocols, a dependency graph for the entire stack can be obtained. Thus, visually you can detect interactions between various protocols in the stack (As shown in Figure 2).

\section{WHICH COMBINATION OF LEVELS OF FACTORS MAXIMIZES NETWORK PERFORMANCE?}

As far as we are aware, the only model proposed to address this question is given in [14]. The method used in

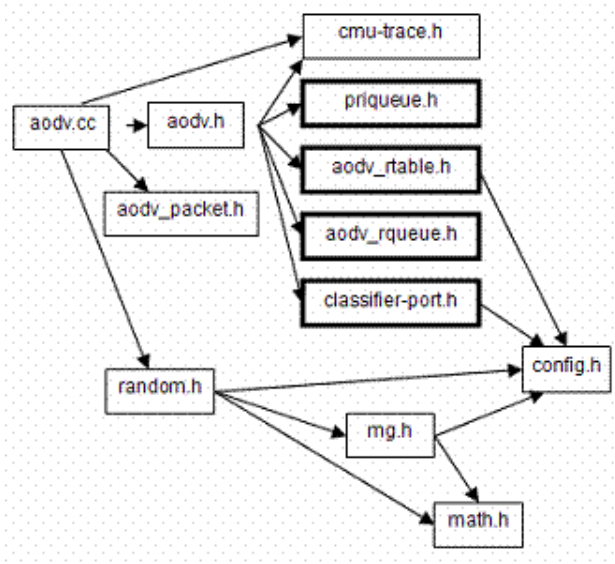

Figure 2. AODV dependency graph.

this paper is the Response Surface Methodology (RSM). RSM finds the specific levels of the relevant factors and produces an optimum performance metric. RSM is a two step methodology.

\subsection{Method of steepest ascent}

The first step finds the region that contains the optimal point. The main assumption made is that the region is flat; thus, technically, we are referring to a hyper plane. Under such assumption we need to estimate only linear regression terms (no quadratic terms and no interaction effects) as shown in Equation 3.

$$
Y=\beta_{0}+\beta_{1} x_{1}+\beta_{2} x_{2}+\ldots+\beta_{p} x_{p},
$$

where $p$ is the number of factors and are the regression coefficients for the factors. The regression coefficients determine the direction of the steepest ascent. For example, suppose we have two factors and two levels; we can then perform a factorial design $\left(2^{2}\right)$ and estimate the plane of the four data points.

\subsection{Method of local exploration}

After a region is identified with the method of steepest ascent, we move to the second step called the method of local exploration. This method probes the surface in greater detail and considers interactions and other non linear terms (usually quadratic). For example, Equation 4 shows the regression terms for factors $x_{1}$ and $x_{2}$.

$$
Y=\beta_{0}+\beta_{1} x_{1}+\beta_{2} x_{2}+\beta_{12} x_{1} x_{2}+\beta_{11} x_{1}^{2}+\beta_{22} x_{2}^{2},
$$

In order to estimate the regression coefficients, i.e., $\beta_{i}$, we need more then a $\left(2^{2}\right)$ factorial design. 


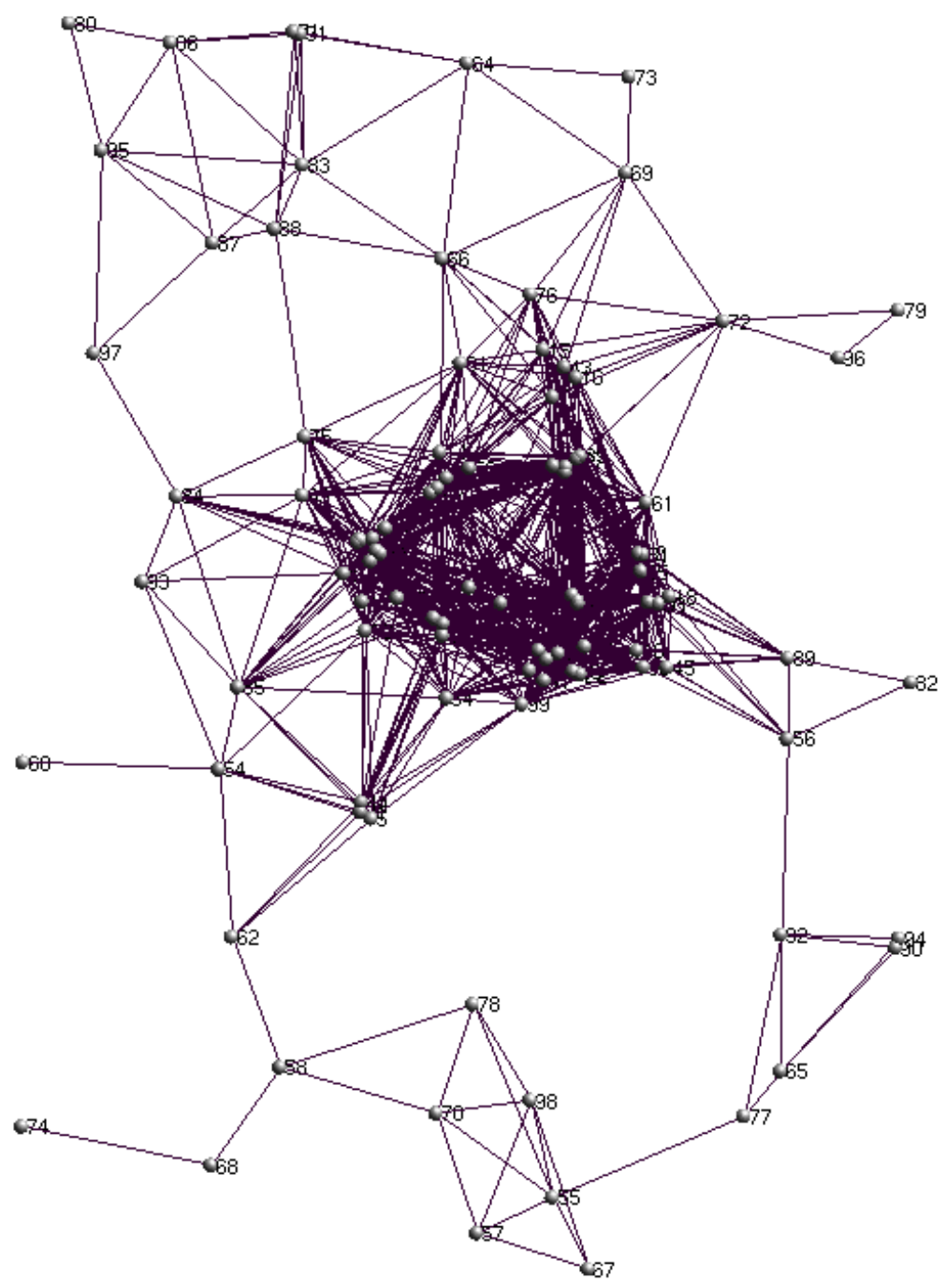

Figure 3. RealMobGen on 100 nodes with Transmission Range 200m.

\section{FRAMEWORK FOR FLEXIBLE AD HOC PROTOCOLS}

RealMobGen (As shown in Figure 3), displays that nodes tend to cluster around access points.

However, the number of access points in a small area only could be very large (See Figure 4). Thus, the first step to design flexible ad hoc protocols, which take into account mobility, is to automatically asses the appropriate number of clusters that the ad hoc protocols should operate into. The problem of determining the number of clusters in unsupervised settings is an NP hard problem. We approach the problem of automatically assessing the number of clusters, as follow. First, we use a supervised method on our design. The supervised method is the well known K-means cluster- ing algorithm. The algorithm takes as input the data points and the number of clusters. We pass the number of clusters incrementally (1, 2, 3, Until a Stopping Criterion is met), since we do not know the number of clusters beforehand.

Second, we use as the stopping criterion the Minimum Description Length (MDL), which was derived by Hansen and $\mathrm{Yu}$ [7]. We implemented the K-means algorithm and the MDL stopping criteria. The experiments in this research were focused on sets of synthetic data that are drawn from spherical Gaussian distributions. Specifically, we demonstrate our findings using that data set, which is composed of eight spherical Gaussian distributions (each with 100 data points).

In this experiment, our goal was to validate through simulations that the implementation will assess eight as the 


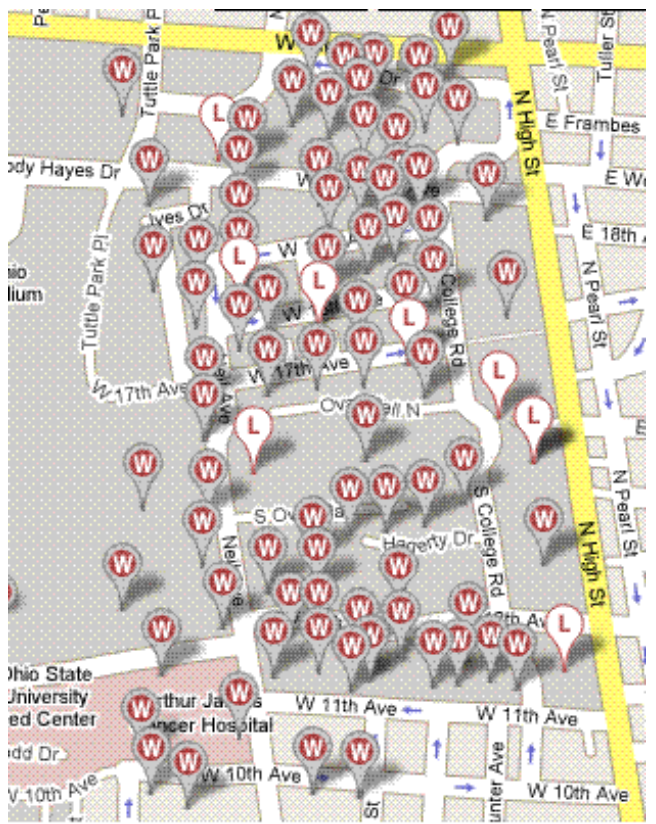

Figure 4. Access Points at a small campus area at Ohio State University.

right number of clusters. We present the simulation results on Figure 5. The smallest value of MDL is selected as a stopping criterion, since the best K-Means linear model is the one that yields the smallest MDL value. The experimental studies show that when the number of clusters is eight the MDL reaches the smallest value, thus assesses eight as the right number of clusters.

The second step, is to design flexible protocols. For example, the de facto ad hoc routing protocols are Ad hoc On-Demand Distance Vector $(A O D V)$ [16] and Dynamic Source Routing $(D S R)$ [3]. Both these protocols are classified as on-demand protocols, due to the fact that a route is discovered only when a packet is needed to be delivered. When a source needs to send data to a destination it issues a route request $(R R E Q)$, until the destination is found. After the destination is found a route reply $(R R E P)$ is send back to the source. If errors occur due to link failures, a route error $(R R E R)$ will be sent back to the source. In addition, two other similarities between the two protocols are the requirement of bidirectional links (due to the need of $R R E P$ to propagate back) and the use of flooding. However, neither of these protocols take into account mobility into the design phase. Instead, we propose to use table driven protocols when within clusters, and either DSR or AODV when between the clusters.

The last step, is to properly assess the information that should be exposed across the layers when taking mobility into account. Specifically, special attention should be
Evaluating k-Means with MDL

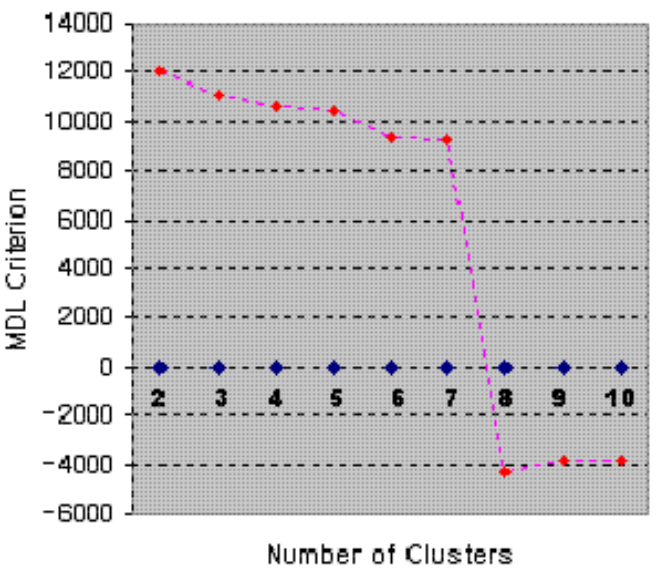

Figure 5. Assessing the Appropriate Number of Clusters.

placed into the Physical, MAC, and Network Layers. The physical and MAC layer should account for the impact of mobility on traffic, as well as, the connectivity graph, while network layer should expose the clusters, as well as, the protocols that it should adjust on the fly between and within clusters.

\section{CONCLUSIONS}

Mobility can offer many benefits into ad hoc networks applications, thus taking it into account in the design phase is very important. In particular, special attention should be placed on designing protocols that make use of the cross layer information and are designed to be flexible and adjust on the fly based on the cluster properties and settings. In the future we plan to assess the correct number of clusters using real data sets, as well as, data that possesses different probability distributions.

\section{References}

[1] L. B. Arta Doci and F. Xhafa, "Mixmobgen - a realistic mixed traffic mobility generator for ad hoc network simulations," in CISIS: Proceedings of the International Conference on Complex, Intelligent, and Software Intensive Systems, 2009.

[2] C. Barrett, A. Marathe, M. V. Marathe, and M. Drozda, "Characterizing the interaction between routing and mac protocols in ad-hoc networks," in $\mathrm{Mo}$ biHoc '02: Proceedings of the 3rd ACM international 
symposium on Mobile ad hoc networking \& computing, 2002, pp. 92-103.

[3] D. M. David Johnson, "Dynamic source routing in ad hoc wireless networks," vol. 353, 1996.

[4] A. Doci and F. Xhafa, "Wit a wireless integrated traffic model," Mobile Information Systems Journal, vol. 4, pp. 219-235, 2008.

[5] Y. Fang and B. McDonald, "Dynamic codeword routing (DCR): a cross-layer approach for performance enhancement of general multi-hop wireless routing," in In Proceedings of IEEE SECON, 2004, pp. 255263.

[6] M. Gerla and D. Raychaudhuri, "Mobility in wireless networks," 2007.

[7] M. Hansen and B. Yu, "Model selection and the principle of minimum description length," Journal of the American Statistical Association, vol. 96, pp. 746774, 2001.

[8] L. V. Hoesel, T. Nieberg, J. Wu, and P. J. M.Havinga, "Prolonging the lifetime of wireless sensor networks by cross-layer interaction," IEEE Wireless Communications, vol. 11, no. 6, pp. 78-86, December 2004.

[9] S. Kurkowski, W. Navidi, and T. Camp, "Discovering factors that affect manet protocol performance," Colorado School of Mines, Tech. Rep. MCS 06-04, June 2006.

[10] J. McNair, T. Tugcu, W. Wang, and J. Xie, "A survey of cross-layer performance enhancements for mobile ip networks," Comput. Netw., vol. 49, no. 2, pp. 119 $146,2005$.

[11] T. Melodia, M. C. Vuran, and D. Pompili, "The state of the art in cross-layer design for wireless sensor networks," in In Proceedings of EuroNGI Workshops on Wireless and Mobility, 2005.

[12] V. Mhatre and C. P. Rosenberg, "Impact of network load on forward link inter-cell interference in cellular data networks," IEEE Transactions on Wireless Communications, vol. 5, no. 12, pp. 3651-3661, December 2006.

[13] V. Mhatre, C. P. Rosenberg, D. Kofman, R. R. Mazumdar, and N. B. Shroff, "Cross-layer design of ad hoc networks for real-time video streaming," IEEE Wireless Communications Magazine, vol. 12, no. 4, pp. 59-65, August 2005.

[14] A. Miller, Subset Selection in Regression, 2nd ed. Chapman and Hall, 2004.
[15] K. Nahm, A. Helmy, and C.-C. J. Kuo, "Tcp over multihop 802.11 networks: issues and performance enhancement," in MobiHoc '05: Proceedings of the 6th ACM international symposium on Mobile ad hoc networking and computing, 2005, pp. 277-287.

[16] C. Perkins and E. Royer, "Ad-hoc on-demand distance vector routing," 1999, pp. 90-100.

[17] D. Perkins, H. Hughes, and C. Owen, "Factors affecting the performance of ad-hoc networks," in In Proceedings of the IEEE International Conference on Communications (ICC), 2002, pp. 2048-2052.

[18] E. Setton, T. Yoo, X. Zhu, A. Goldsmith, and B. Girod, "Cross-layer design of ad hoc networks for realtime video streaming," IEEE Wireless Communications Magazine, vol. 12, no. 4, pp. 59-65, August 2005.

[19] V. Srivastava and M. Motani, "Cross-layer design: a survey and the road ahead," IEEE Communications Magazine, vol. 43, no. 12, pp. 112-119, December 2005.

[20] M. Takai, J. Martin, and R. Bagrodia, "Effects of wireless physical layer modeling in mobile ad hoc networks," in MobiHoc '01: Proceedings of the 2nd ACM international symposium on Mobile ad hoc networking \& computing, 2001, pp. 87-94.

[21] M. W. Totaro and D. D. Perkins, "Using statistical design of experiments for analyzing mobile ad hoc networks," in MSWiM '05: Proceedings of the 8th ACM international symposium on Modeling, analysis and simulation of wireless and mobile systems, 2005, pp. 159-168.

[22] K. Vadde and V. Syrotiuk, "Factor interaction on service delivery in mobile ad hoc networks," IEEE Journal on Selected Areas in Communications, vol. 22, no. 7, pp. 1335-1346, 2004.

[23] M. C. Vuran, V. B. Gungor, and O. B. Akan, "On the interdependency of congestion and contention in wireless sensor networks," in In Proceedings of SENMETRICS, July 2005, pp. 136-147.

[24] C. Walsh, A. Doci, and T. Camp, "A call to arms: it's time for real mobility models," vol. 12, no. 1, 2008, pp. 34-36.

[25] W. Yuan, K.Nahrstedt, S. Adve, D. Jones, and R. Kravets, "GRACE-1: cross-layer adaptation for multimedia quality and battery energy," IEEE Transactions on Mobile Computing, vol. 5, no. 7, pp. 799815 , July 2006. 